\title{
UN TESTIMONIO DE COMUNICACIÓN ENTRE LAS ESCUELAS JURISPRUDENCIALES ROMANAS
}

\author{
Elvira ARIZA TIRADOS \\ Departamento de Derecho romano e Historia del Derecho \\ Facultad de Derecho. Universidad Complutense de Madrid \\ eariza@ucm.es
}

\section{EL PROBLEMA DE LA SUPUESTA SEPARACIÓN ENTRE LAS ESCUELAS JURISPRUDENCIALES ROMANAS}

La idea de la investigación que enmarca este trabajo partió de unas lecturas acerca del jurista Nerva, que me condujeron a la obra de Gian Luigi FALCHI, Le controversie tra proculiani e sabiniani (Milán, 1981). En esta obra el autor reúne los diferentes criterios que tradicionalmente ha buscado la doctrina para diferenciar las escuelas jurisprudenciales romanas y aporta su visión personal acerca de los mismos. Se trata, sin duda, del mejor ejemplo doctrinal de esta línea de investigación, como consecuencia de la cual hoy en día se tiende a considerar las escuelas jurisprudenciales romanas como grupos profesionales netamente separados ${ }^{1}$.

1 Sobre las escuelas jurisprudenciales romanas y los criterios de diferenciación elaborados de las mismas, vid. H. E. DiRKSEN, Beiträge zur Kunde des römischen Rechts, vol. I, Abhandlung: Über die Schulen der römischen Juristen, Leipzig, Hinrichs, 1825; A. PERNICE, Marcus Antistius Labeo. Das römische Privatrecht, Halle, Buchhandlung des Waisenhauses, 1873; M. SchanZ, «Die Analogisten und Anomalisten im römischen Recht», Philologus, vol. 42 (1884), pp. 309-318; O. KARLOWA, Römische Rechtsgeschichte, vol. I, Leipzig, Veit \& comp., 1885, pp. 657 y ss.; M. VoIgT, Römische Rechtsgeschichte, vol. II, Stuttgart, Cotta, 1899, pp. 222 y ss.; G. F. Puchta, Cursus der Institutionem, Leipzig, Breitkopf \& Härtel, 1893, pp. 430 y ss.; P. SoKOLOwsKI, «Die Lehre von der Specification», ZSS, vol. 17 (1896), pp. 252-311; G. BAVIERA, Le due scuole dei giureconsulti romani, Roma, «L'Erma» di Bretschneider, 1970; E. BETTI, «Sul valore dogmatico della categoria contrahere in giuristi proculiani e sabiniani», BIDR, vol. 28 (1915), pp. 3-96; C. G. Bruns, A. Pernice y O. Lenel, «Geschichte und Quellen des römisches Rechts», en Holtzendorff Enzyklopädie, vol. I, Leipzig-Berlin-München, Duncker \& Humblot-J. Guttentag, 1915, pp. 133 y ss.; G. G. ARCHI, L'«Epitome Gai». Studio sul tardo Diritto romano in Occidente, Milano, Jovene, 1937; S. Riccobono, «La giurisprudenza classica come fattore di evoluzione nel Diritto roma- 
Esta visión tradicional de las escuelas jurisprudenciales tiene, en efecto, un fundamento sólido en las fuentes jurídicas romanas: en el Digesto de Justiniano encontramos noticias de las escuelas de mano de Pomponio, que, como es sabido, fue tanto jurista como historiador romano durante el siglo II d. C. Será él quien nos acerque en su obra Enchiridion al nacimiento y posterior desarrollo de las escuelas que él denominaba sectae. Destacan entre sus textos los siguientes:

no», en G. G. Archi (ed.), Scritti di Diritto romano in onore di Contardo Ferrini, Milano, Ulrico Hoepli Editore, 1946, pp. 2-80; F. Schulz, History of Roman Legal Science, Oxford, Clarendon Press, 1946, pp. 214 y ss.; A. Magdelain, «Ius Respondendi», RHD, vol. 28 (1950), pp. 1-22; M. KASER, Römische Rechtsgeschichte, 2. ${ }^{a}$ ed., Göttingen, Vandenhoeck \& Ruprecht, 1971, pp. 201 y ss.; G. CRIFÒ, «Il suicidio di Cocceio Nerva "pater" e i suoi riflessi sui problemi del quasi usufrutto», en Studi in onore di Gaetano Scherillo, vol. I, Milano, Istituto Editoriale Cisalpino La Goliardica, 1972, pp. 427-440; P. STEIN, «The Two Schools of Jurists in the Early Roman Principate», CLJ, vol. 31 (1972), pp. 8-31; M. BRETone, Linee dell'Enchiridion di Pomponio, Torino, Giappichelli, 1974; C. A. Cannata, La giurisprudenza romana, Torino, Giappichelli, 1974; D. LIEBS, «Rechtsschulen und Rechtsunterricht im Prinzipat», ANRW, t. II, vol. 15 (1976), pp. 197-286; V. Arangio-Ruiz, Sto-

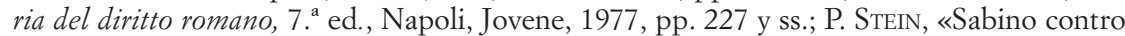
Labeone», BIDR, vol. 50 (1977), pp. 55-67; M. TALAMANCA, «Per la storia della giurisprudenza romana», BIDR, vol. 80 (1977), pp. 195-344; S. TONDO, «Note esegetiche sulla giurisprudenza romana», Iura, vol. 30 (1979), pp. 34-77; G. L. FALCHI, Le controversie tra Sabiniani e Proculiani, Milano, Giuffrè, 1981; D. NÖRR, «I giuristi romani: tradizionalismo o progresso?», BIDR, vol. 23 (1981), pp. 9-33; M. BRETONE, Tecniche e ideologie dei giuristi romani, Napoli, Edizioni Scientifiche Italiane, 1982; W. SELB, «Formulare Analogien in actiones utiles und actiones in factum am Beispiel Julians», en Studi in onore di Arnaldo Biscardi, vol. III, Milano, Istituto Editoriale Cisalpino La Goliardica, 1982, pp. 315-350; A. Guarino, Le ragioni del giurista, Napoli, Jovene, 1983; M. G. SCACHetTI, «Diffrenze di método fra sabiniani e proculiani», en Studi Biscardi, vol. V, Milano, Istituto Editoriale Cisalpino La Goliardica, 1984; M. D’ORTA, «Per una storia della cultura dei giuristi repubblicani», BIDR, vol. 29 (1987), pp. 221-284; T. GIARO, «Dogmatische Wahrheit und Zeitlosigkeit in der römischen Jurisprudenz», BIDR, vol. 29 (1987), pp. 1-104; A. GUARINO, Storia del Diritto romano, 7. a ed., Napoli, Jovene, 1987, pp. 491 y ss.; M. D’ORTA, La giurisprudenza tra repubblica e principato, Napoli, Edizioni Scientifiche Italiane, 1990; P. STEIN, «Interpretation and Legal Reasoning in Roman Law», CKLR, vol. 70 (1995), pp. 1539 1556; F. GALLO, «Sulle tracce di indirizzi sabiniani e proculiani nella materia contrattuale»; A. Mantello, «Un'etica per il giurista? Profili d'interpretazione giurisprudenziale nel primo Principato», y P. Stein, «Le scuole», los tres en D. Mantovani (ed.), Per la storia del pensiero giuridico romano, Torino, Giappichelli, 1996, pp. 15-38, pp. 147-182 y pp. 1-13, respectivamente; E. STOLFI, «Il modello delle scuole in Pomponio e Gaio», SDHI, vol. 63 (1997), pp. 1-106; W. KunKeL, Herkunft und soziale Stellung der Römischen Juristen, 2. ${ }^{a}$ ed., Köln-Weimar-Wien, Böhlau, 2001; P. SteIN, «I giuristi e le scuole», en F. Milazzo (ed.), Ius controversum $e$ auctoritas principis. Giuristi principe e diritto nel primo impero, Napoli, Edizioni Scientifiche Italiane, 2003, pp. 299-311; W. Kunkel, Römische Rechtsgeschichte, 14. ${ }^{a}$ ed. revisada por M. SCHERMEIER, Köln-Weimar-Wien, UTB, 2005, pp. 151 y ss.; J. DE Churruca y R. MentxaKa, Introducción histórica al Derecho romano, 9. ${ }^{a}$ ed, Bilbao, Universidad de Deusto, 2007, pp. 156 y ss., y A. Fernández Barreiro y J. Paricio, Historia del Derecho romano y su recepción europea, 11. ${ }^{a}$ ed., Madrid, Marcial Pons, 2010, pp. 112 y ss. 
«Hi duo primi veluti diversas sectas fecerunt: nam Ateius Capito in bis, quae ei tradita fuerant perseverabat, Labeo ingenii qualitate et fiducia doctrinae, qui et ceteris operis sapientiae operam dederat, plurima innovare instituit» [D. 1,2,2,47 (Pomp. enchir. sing.)].

«Et ita Ateio Capitoni Massurius Sabinus successit, Labeoni Nerva, qui adbuc eas dissensiones auxerunt, bic etiam Nerva Caesari familiarissimus fuit». [D. 1,2,2,48 (Pomp. enchir. sing.)].

«Appellatique sunt partim Cassiani, partim Proculiani, quae origo a Capitone et Labeone coeperat» [D. 1,2,2,52 (Pomp. enchir. sing.)].

Como se puede ver, Pomponio nos revela, en primer lugar, que Labeón y Capitón crearon «como dos escuelas» (veluti diversas sectas); a continuación, que estos fueron sucedidos por Nerva y Masurio Sabino, respectivamente; por fin, que estos últimos «aumentaron las disensiones» (dissensiones auxerunt), dando a entender que no hacían sino profundizar en las diferencias que ya los fundadores de las escuelas habrían marcado.

Desde otro punto de vista, Pomponio también nos habla de la personalidad de los fundadores mismos, aportando quizá datos relevantes que pudieran explicar la diferencia entre ambas escuelas: por un lado, Capitón, quien, según Pomponio, perseveró en la tradición (in his, quae ei tradita fuerant perseverabat); por el otro, Labeón, hombre de vastísima cultura y gran ingenio (ingenii qualitate et fiducia doctrinae) que innovó en muchos aspectos del Derecho (plurima innovare instituit). Como queda patente, de esta obra de carácter histórico se pueden obtener ya lo que parecen criterios de distinción entre las dos sectae.

En el estudio de las escuelas jurisprudenciales romanas no se puede obviar, por otra parte, el testimonio de las Instituciones de Gayo. El papel que juega el jurista en la comprensión de las escuelas es vital, ya que es él quien aporta el mayor número de testimonios sobre controversias entre ambos grupos. Gayo, que se consideraba «sabiniano» en una época en la que las escuelas parecen estar ya totalmente superadas, se refiere a sus «maestros» de una forma muy personal. Así, en ocasiones habla de «nuestros preceptores» (nostri praeceptores) y de los autores «de la otra escuela» (diversae scholae auctores), y, en otras, nos da valiosa información sobre juristas en concreto, bien sean de una u otra escuela:

«Item si quis sub ea condicione stipuletur, quae existere non potest, veluti si digito caelum tetigerit, inutilis est stipulatio. Sed legatum sub impossibili condicione relictum nostri praeceptores proinde deberi putant, ac si sine 
condicione relictum esset; diversae scholae auctores nibilo minus legatum inutile existimant quam stipulationem. Et sane vix idonea diversitatis ratio reddi potest». (Gai. 3,98).

Recapitulando: Pomponio se refiere a «casianos» y «proculianos», mientras que Gayo habla de «preceptores» de una u otra escuela.

Pero ahí empieza el problema: la doctrina se halla dividida en el modo mismo de entender el concepto de «escuela jurídica». Así, de un lado, podemos encontrar la posición del sector más tradicional de la doctrina que, siguiendo la estela que dejara Pomponio, ve las escuelas como instituciones independientes que construyeron durante siglos unas características que las distinguían entre sí.

Pero, ¿cuáles eran estas características diferenciadoras? Pomponio solo menciona, muy superficialmente, el carácter más o menos conservador o innovador de sus fundadores. Por ello, esta parte de la doctrina se ha centrado en estudiar los numerosísimos ejemplos de disputas doctrinales entre representantes de ambas escuelas, para intentar dilucidar la metodología jurídica propia de cada una de ellas. Así, el denominado «ius controversum» —es decir, el derecho «discutido» o «controvertido»- se ha convertido en el material de trabajo fundamental a partir del cual se han intentado destilar los distintos criterios de clasificación; criterios que esta parte de la doctrina ha elaborado a la búsqueda de un concepto de escuela de líneas puras.

Resulta imposible enumerar, en los límites de este trabajo, todos y cada uno de los criterios ${ }^{2}$ que se han elaborado, que abarcan puntos de vista tan variados como el filosófico, el metodológico, el político, etc.; sin embargo, lo que cabe destacar es que hasta el momento no ha sido posible encontrar un concepto de escuela unitario a partir de ellos, ya que los propios autores que defienden la separación la ven de forma diferente, en ocasiones incluso contradictoria, lo que los ha llevado a numerosas discusiones doctrinales.

\footnotetext{
${ }^{2}$ A este respecto una de las obras de referencia que analizan los criterios doctrinales de forma sistemática es, en efecto, el ya mencionado G. L. FALCHI, Le controversie tra Sabiniani..., op. cit. El autor italiano, que comienza su obra criticando la poca profundidad con la que se han estudiado las controversias entre las escuelas jurisprudenciales (p. 3), expone en la primera parte los criterios que se han elaborado y los autores que los han defendido, para después acometer el análisis de las fuentes que presentan las discusiones entre proculianos y casianos. La obra ha sido un pilar fundamental en la elaboración del estado de la cuestión al reunir la opinión de gran parte de la doctrina de una manera exhaustiva y sistematizada.
} 
De hecho, esta falta de unidad (criticada por autores como Schulz ${ }^{3}$ o Arangio-Ruiz ${ }^{4}$, que ven en ello un fracaso de esta interpretación de las fuentes) servirá a otra parte de la doctrina — que no contempla las escuelas como bloques opuestos desde el punto de vista de la metodología jurídica- para reflexionar acerca de las sectae desde otros puntos de vista. En efecto, la teoría crítica no ve una diferenciación tan clara en lo que a metodología se refiere, llegando autores como Schulz a considerar estas supuestas diferencias entre escuelas meros «points of detail $\rangle^{5}$. Según los críticos, se debería concebir las escuelas más bien como centros diversos en los que juristas en formación aprendían de los juristas en ejercicio de un arte único, el del Derecho. Así, si bien la adhesión a una escuela u otra efectivamente existiría, tal y como parece deducirse de las fuentes, esta se produciría, según estos autores, por razones no de tipo metodológico-profesional, sino de orden más bien personal. Guarino ${ }^{6}$, por ejemplo, hace referencia tanto a razones familiares ${ }^{7}$ como a la pietas hacia el maestro, a modo de ejemplos de elementos aglutinadores que habrían mantenido las sectae unidas durante largas generaciones.

Pero, dicho todo lo anterior, no se debe olvidar que las fuentes plantean, además, un obstáculo de base que condiciona a nivel exegético la interpretación de los testimonios conservados. En efecto, en el Digesto encontramos no solo alusiones genéricas a proculianos y sabinianos, sino también numerosas referencias a juristas que se expresan de modo individual. Por ello, desde siempre se ha suscitado el interés por estudiar las controversias entre juristas, para de este modo intentar comprender si en sus respuestas se aplicaban criterios metodológicos propios - los cuales definirían las opiniones propias de cada escuela y, por tanto, su esencia y personalidad- o si más bien las controversias documentadas se trataban en realidad tan solo de opiniones adoptadas por juristas de forma particular, si bien dentro de sus respectivas stationes.

En este sentido, Dirksen ${ }^{8}$ establece ya una división de las opiniones jurídicas que se emitían en las controversias, las cuales clasifica

${ }^{3}$ F. Schulz, History of Roman Legal..., op. cit., p. 122.

${ }^{4}$ V. Arangio-Ruiz, Storia del diritto..., op. cit., p. 341.

${ }^{5}$ F. Schulz, History of Roman Legal..., op. cit., p. 122.

${ }^{6}$ A. Guarino, Storia del Diritto..., op. cit., p. 301.

7 Se sabe, por ejemplo, que la esposa de Labeón pertenecía a la familia de los Neracios, es decir, emparentada con quien décadas más tarde sería uno de los últimos jefes de la escuela proculiana, Neracio Prisco. Sobre esto vid., por todos, W. KunKel, Herkunft und soziale Stellung..., op. cit., p. 114.

${ }^{8}$ H. E. DiRKSEN, Beiträge zur Kunde..., op. cit., pp. 11 y ss. 
como Schulmeinungen (opiniones de escuela) y Privatmeinungen (opiniones personales de juristas que no habrían sido seguidas por todos los jurisprudentes y, por tanto, no habrían llegado a convertirse en opinión de escuela).

Por su parte, Baviera, en su monografía dedicada a las escuelas de juristas ${ }^{9}$, delimita lo que considera como Schulmeinung y lo que entiende por Privatmeinung. Siguiendo la clasificación de Dirksen, Baviera cree que puede afirmarse que las Schulmeinungen surgen en realidad como una discusión entre dos juristas con carácter de Privatmeinung, hasta que los auditores, al hacerse eco de estas y adoptarlas, las convierten en opinión de escuela. El resto de discusiones no podrían considerarse como Schulmeinungen al limitarse a simples discrepancias entre dos o tres juristas y que, por tanto, no alcanzarían tal grado.

Respecto al caso concreto de Gayo, Baviera no duda de que las controversias que cita sean verdaderas opiniones de escuela. En los casos en los que para hablar de las escuelas el jurista utiliza las expresiones «nostri praeceptores» o «diversae scholae auctores», la clasificación de las distintas opiniones es claramente la de Schulmeinung. También lo cree de este modo en lo que se refiere a las controversias en las que Gayo hace referencia a juristas en concreto. Sabino, Casio, Nerva y Próculo eran juristas dotados del ius publice respondendi, capaces, por tanto, de influir de forma determinante con sus decisiones en el Derecho vigente ${ }^{10}$. El hecho de que Gayo solo los nombrara a ellos puede interpretarse, según Baviera, como indicio de que el resto de los praeceptores y de los auditores seguían estas opiniones. De ahí que Baviera no dude al considerar las disensiones citadas por Gayo como verdaderas Schulmeinungen.

Como se puede observar, no obstante, existe división de opiniones en cuanto a los criterios que identifican y separan a cada escuela. En general, la doctrina coincide en una visión de las escuelas como entes separados con rasgos diferenciales propios.

Sin embargo, de una lectura de las fuentes jurídicas desde una óptica diferente - esto es, de los textos que las corrientes doctrinales arriba mencionadas han dejado tradicionalmente de lado- se pueden extraer numerosos testimonios que permiten, en su conjunto, ver las escuelas con una perspectiva novedosa. Se trata de testimonios que parecen revelar una realidad menos tajante, un ambiente de comunicación entre escuelas cuya

\footnotetext{
${ }^{9}$ G. BAVIERA, Le due scuole dei giureconsulti.., op. cit., pp. 38 y ss.

${ }^{10}$ Ibid., pp. 38 y ss.
} 
constatación obligaría, si no a desmentir la visión tradicional de la neta separación entre escuelas, sí al menos a matizarla. Podría así someterse a revisión, desde esta óptica, la — por otra parte, muy meritoria- obra de Falchi, aportando el estudio de los testimonios dejados al margen en la tradicional búsqueda de pruebas de una separación clara entre las escuelas jurisprudenciales romanas.

En este momento me encuentro realizando una amplia investigación que incluye la detección y análisis de los mencionados testimonios. Para este trabajo, sin embargo — debido a las lógicas limitaciones de espacio-, se propone analizar con detalle uno de estos testimonios que permite ilustrar las posibilidades exegéticas de esta metodología de trabajo.

\section{MATERIALES PARA LA REVISIÓN DE LA DOCTRINA TRADICIONAL: UN TESTIMONIO DE COMUNICACIÓN ENTRE ESCUELAS}

El testimonio escogido para la presente exégesis se encuentra en un fragmento atribuido al jurista Paulo:

«Rerum amotarum iudicium singulare introductum est adversus eam quae uxor fuit, quia non placuit cum ea furti agere posse: quibusdam existimantibus ne quidem furtum eam facere, ut Nerva Cassio, quia societas vitae quodammodo dominam eam faceret: aliis, ut Sabino et Proculo, furto quidem eam facere, sicut filia patri faciat, sed furti non esse actionem constituto iure, in qua sententia et Iulianus rectissime est» [D. 25,2,1 (Paul. 7 Sab.)].

La exégesis de D. 25,2,1 debe comenzar por el estudio de la cuestión jurídica que el texto trata. Dicha discusión se da en torno a la justificación de la introducción en el ordenamiento jurídico de la actio rerum amotarum en sustitución de la actio furti cuando la mujer sustrae bienes del que había sido su marido.

Los juristas que intervienen en esta discusión son Sabino, Casio, Nerva y Próculo, quienes se posicionan formando un peculiar cruce. Se debe recordar que Sabino y Nerva eran los discípulos de los fundadores de las escuelas, es decir, Capitón (de la escuela casiana o sabiniana) y Labeón (de la escuela proculiana), respectivamente. A su vez, Casio fue discípulo de Sabino y Próculo de Nerva. En el texto cada alumno se posiciona del lado del maestro contrario, de ahí lo especial del fragmento. 
Un texto aparentemente tan fuera de lo común ha de tratarse con sumo cuidado, a pesar de que autores como Wacke ${ }^{11}$, que de hecho es de los poquísimos autores que advierte el cruce, no cree que haya porqué dudar de su autenticidad ${ }^{12}$.

Entremos en detalles. Nerva y Casio son los primeros prudentes mencionados por Paulo en su texto. Y a ellos debemos el primero de los dos razonamientos que se exponen en el mismo para justificar la acción. Nerva y Casio afirman que la fundamentación de la actio rerum amotarum reside en la condición de dueña de la que ha sido esposa. Condición que, de algún modo (quodammodo), le otorga la convivencia con su esposo durante el matrimonio. La esposa, técnicamente, no hurtaría, ya que, según estos dos juristas, no se pueden hurtar las cosas que son de nuestra propiedad. De esta forma, al no cometerse hurto y no darse, por tanto, el delito, no procedería la interposición de la actio furti y se propondría el uso de una acción alternativa como es la actio rerum amotarum.

$\mathrm{El}$ argumento que esgrimen ambos juristas parece ambiguo, ya que no se funda claramente en un derecho real stricto sensu; este razonamiento, al límite del Derecho establecido, podría hacernos pensar que esta opinión no es sino un caso aislado en la línea general de la jurisprudencia $^{13}$. Sin embargo, el recurso al criterio del quodammodo, al que recurren Nerva y Casio en su exposición, no le es desconocido a la doctrina, ya que se ha constatado que los juristas lo han utilizado también en otros $\operatorname{casos}^{14}$. Existe, por otro lado, una discusión de corte interpola-

11 A. WACKE, Actio rerum amotarum, Köln, Böhlau, 1963, p. 90.

${ }^{12}$ La problemática transmisión de los textos jurídicos clásicos exige una metodología específica que, como es sabido, abarca también la crítica interpolacionística [vid. sobre ello M. KASER y F. WIEACKER, Las interpolaciones en las fuentes juridicas romanas, Granada, Comares, 1998; traducción de J. M. ${ }^{a}$ Coma y G. Gallenkamp de los dos estudios fundamentales Ein Jahrbundert Interpolationenforschung an den römischen Rechtsquellen, Köln, 1986, del primero, y «Textkritik und Sachforshung», SZ, vol. 91 (1974), del segundo] y la crítica textual. Mientras la primera será aludida en la medida pertinente en este trabajo, en cuestiones de crítica textual me apoyo en la edición de T. MommSen, P. KRÜGer, R. Schöll y W. Kroll del Corpus Iuris Civilis (diferentes ediciones según el volumen), que no arrojaban variantes significativas para ninguno de los textos aquí estudiados.

${ }^{13}$ La consideración de la esposa como propietaria «de algún modo» de los bienes del marido, explica Wacke, solo tenía validez para los juristas que la defendían en el caso del matrimonio cum manu, puesto que permanecía loco filiae, y así como a los hijos, también a ella se la consideraba en vida del paterfamilias «quodammodo domina». Vid. A. WACKE, Actio rerum amotarum, op. cit., p. 92.

${ }_{14}$ Vid, por ejemplo, D. 28,2,11, en el que se habla del filius familias como heredero que, antes incluso de la transmisión de la herencia, es dueño «de algún modo» de las cosas del padre de familia, «qui etiam vivo patre quodammodo domini existimantur». 
cionístico respecto al uso del adverbio quodammodo entre Albanese ${ }^{15} \mathrm{y}$ Solazzi ${ }^{16}$ que en cualquier caso excede el asunto a tratar, ya que parece que el núcleo del texto - es decir, el cruce de opiniones entre juristas de escuelas diferentes, que es el aspecto más relevante- es indudablemente genuino.

Sabino y Próculo, por su parte, defendían la idea de que la mujer cometía técnicamente hurto, al igual que la hija puede cometerlo con las cosas de su padre. Sin embargo, creían que, en aras al honor del matrimonio, la acción infamante de hurto no debía darse contra la que había sido esposa, tal y como se explica en el fragmento siguiente:

«Nam in honorem matrimonii turpis actio adversus uxorem negatur» [D. 25.2.2. (Gai. ed. praet., tit. de re iud.)].

Según Gayo, que es quien nos transmite el fundamento de la interpretación de Sabino y Próculo, estos no creían correcto que se aplicara la acción de hurto no porque no consideraran que en efecto el hurto se habría producido, sino en consideración al bonor del matrimonio, concepto que gozaba de un gran peso social y moral en la Roma clásica.

Ahora bien, no se debe olvidar que en Roma se dieron dos tipos muy diferentes de matrimonio, cada uno con una regulación jurídica distinta, que afectaba también al ámbito procesal. Así, en el matrimonio cum manu la interposición de cualquier acción entre los cónyuges estaba excluida, ya fuesen acciones infamantes o no; esta exclusión, por el contrario, no existía en los matrimonios sine manu.

Por tanto, parece lógico preguntarse si la afirmación de Sabino y Próculo se refería a ambas clases de matrimonio. En principio cabría pensar que en un matrimonio sine manu una acción como la de hurto pudiera interponerse, no habiendo impedimento jurídico alguno, a no ser el de orden ético-social como el aportado por el texto de Gayo en D. 25,2,2. Pero en opinión de Wacke ${ }^{17}$, la exclusión de la acción de hurto en el caso de los matrimonios sine manu se basaría más bien en un intento de equiparar a la uxor sine manu y a la uxor cum manu en el derecho a que no se litigase contra ella en el supuesto concreto de que hubiera de observarse la actio furti, ya que, para el resto de las acciones, incluso las infamantes, no se seguía esta pauta. Así, salvo en casos como el aquí estudiado, la prohi-

\footnotetext{
15 M. Albanese, «Succesione ereditaria», Annali di Palermo, vol. 20 (1949), pp. 218-249.

${ }^{16}$ S. SolazZI, «Quodammodo nelle Istituzioni di Gaio», SDHI, vol. 19 (1953), pp. 104 -133.

17 A. WACKE, Actio rerum amotarum, op. cit., p. 93.
} 
bición total de interponer acciones entre esposos seguiría siendo exclusiva de los matrimonios cum manu.

Una vez explicados los términos de la controversia, volvamos al tema que a este trabajo interesa: el cruce de opiniones entre juristas de escuelas contrarias. El cruce documentado en D. 25,2,1 tiene dos aspectos importantes que deben ser resaltados:

a) De un lado, el hecho de que ambas escuelas recurran a una misma metodología —es decir, a un mismo criterio de interpretatiopara resolver una cuestión. Y así, Nerva y Casio, juristas de escuelas contrarias, recurren a una ficción jurídica, mientras que Sabino y Próculo, también de escuelas contrarias, optan por alegar la validez de una convención social y ética como fundamento de una solución jurídica. Así las cosas, un texto semejante va contra la parte de la doctrina que define las escuelas basándose en diferencias metodológicas al no cuadrar en ninguno de los criterios que este sector propone.

b) De otro lado, nos encontramos con que los discípulos no solo se posicionan junto a los maestros de la escuela contraria, sino que van en contra de lo establecido por sus propios maestros. De este modo, viene también a matizarse el concepto de pietas que hasta ahora se ha manejado, que a la vista de testimonios como los que nos ocupan parece algo más frágil.

De hecho, una exégesis de D. 25,2,1 desde una perspectiva más amplia - como puede ser la de su análisis sistemático o el estudio de su contexto histórico- obliga a reflexionar algo más sobre esta cuestión.

Así, resulta especialmente reveladora la ubicación sistemática del texto en el Digesto, puesto que se trata del fragmento que abre precisamente el libro dedicado a la actio rerum amotarum; eso permite deducir que los compiladores no encontraban óbice alguno en el hecho de que un texto tan fundamental sobre la exclusión de la actio furti dentro del matrimonio se base en un cruce de opiniones entre maestros y discípulos de escuelas diferentes. Se trata de una circunstancia que solo a nuestros ojos, no a los de ellos evidentemente, resulta tan singular.

Por otro lado, un análisis del contexto histórico del texto -atendiendo a la antigüedad de la acción - debe ser aplicado también, pues el elemento cronológico podría haber influido en la peculiaridad de la discusión documentada en el texto. En efecto, cabría suponer que en el caso de una acción de reciente introducción, las opiniones sobre su valor y función no 
habrían tenido tiempo aún de cristalizar, produciéndose así la controversia entre juristas. Si tenemos en cuenta que la referencia más antigua de un jurista hablando de dicha acción nos lleva a Ofilio, predecesor de Capitón y que vivió a finales del periodo republicano, podríamos situar la creación de la actio rerum amotarum probablemente en un tiempo no demasiado lejano del de los juristas que protagonizan el texto analizado. Esta hipótesis la plantea también Burdese ${ }^{18}$, quien, en efecto, sitúa la creación de la actio en la segunda mitad del último siglo de la República. De ser esto así, podríamos pensar que la reciente creación de la actio no habría dado tiempo para que se formase una Schulmeinung y, por ello, en el texto los juristas estarían aún emitiendo sus opiniones solo de forma personal.

No se trataría en dicho supuesto de un verdadero «cruce de escuelas», sino de un testimonio muy interesante que documentaría el trabajo individual previo de los juristas antes de la formación de una opinión común en cada escuela. Sin embargo, tanto la común técnica interpretativa entre juristas de diferente escuela como la oposición al propio maestro hacen difícil imaginar una futura cristalización de dos criterios de escuela diferenciados. Aquí sería relevante recordar el criterio de exégesis sistemática arriba aplicado: el hecho de que los compiladores seleccionaran este texto para abrir el correspondiente título revelaría que, al final, no se habría formado una opinión de escuela que pudiera citarse.

\section{RESULTADOS Y PERSPECTIVAS METODOLÓGICAS}

De todo lo expuesto hasta ahora se puede concluir lo siguiente.

Lo planteado en D. 25,2,1 es un ejemplo de cómo los juristas de diferentes escuelas podían no solo servirse de idénticas metodologías para solucionar una cuestión de Derecho, sino además convenir con los jurisconsultos de las escuelas contrarias o incluso oponerse a los de las propias, aunque las opiniones a batir fuesen las de sus propios maestros.

Se pone, por tanto, en cuestión, en primer lugar, el criterio doctrinal tradicional que funda la diferencia entre escuelas jurisprudenciales en el uso de herramientas metodológicas distintas; por otra parte, resquebraja también el concepto de pietas que la parte crítica de la doctrina defendía como elemento aglutinador alternativo de las escuelas. De hecho, si bien podría pensarse que la reverencia hacia la figura del preceptor pudiera

18 A. BuRDESE, «Recensión a A. Wacke, Actio rerum amotarum», Iura, vol. 15 (1964), p. 328. 
coartar la independencia de los juristas a la hora de emitir opiniones contrarias a las de sus maestros, no solo este texto, sino muchos otros de los textos analizados en el marco de la investigación que está realizando quien esto suscribe, demuestran que ni siquiera los más ilustres juristas se libraron de las críticas de sus discípulos.

Testimonios tan valiosos como el que nos transmite D. 25,2,1 han quedado al margen de la atención de la doctrina tradicional, pues esta, al buscar un concepto de escuela con unos límites puros, ha elaborado unos criterios de diferenciación para lo cual ha realizado una doble criba de las fuentes: como ya se ha mencionado, se ha partido, en primer lugar, de un concepto de ius controversum bastante restringido, en el que las controversias estudiadas solo serán consideradas controversias de escuela como tal si la discusión se genera entre juristas de escuelas diferentes; con esto se dejaban ya fuera de su estudio discusiones desarrolladas dentro de la propia escuela.

En segundo lugar, en el afán de acotar aún más el terreno del que extraer las características que delimitasen las escuelas, la distinción entre Schulmeinung y Privatmeinung ha provocado que textos que documentaban controversias entre juristas de escuelas diferentes tampoco se admitieran como testimonios de controversias de escuela, si alguna de ellas no podía considerarse como una Schulmeinung.

El resultado de estos criterios metodológicos dejó fuera del foco de atención numerosos testimonios como el que ha centrado este trabajo, que podrían haber proporcionado un punto de vista más amplio de las escuelas y su actividad.

Pero dado que en ningún caso la revisión de estas corrientes exegéticas tradicionales puede fundamentarse en la exégesis de un solo texto, la investigación en la que se enmarca este trabajo abarca un corpus bastante extenso, con el que se pretende obtener una perspectiva del concepto de escuela más matizada que el que se ha venido manejando hasta el momento. La labor heurística para la selección de textos se ha estructurado en bloques temáticos que rompen los criterios de diferenciación tradicionales. Así, se han localizado testimonios en los que los alumnos matizan las opiniones de sus maestros, textos en los que incluso se rechaza la opinión de estos o pasajes en los que los juristas convienen con los juristas de la otra escuela, en ocasiones — como acabamos de ver en D. 25,2,1—, en contra incluso de la opinión de miembros de su misma escuela.

Los testimonios que conforman el corpus, por otra parte, se refieren a materias jurídicas muy diversas, que van desde las sucesiones a los derechos reales o el Derecho procesal. En otras palabras, ya solo con la sis- 
tematización de los testimonios identificados se puede apreciar que la materia sobre la que versan las controversias no influye para que la comunicación entre escuelas o las discrepancias en el seno de las mismas se produzcan continuamente.

Si bien la investigación está aún en proceso de realización, los textos ya estudiados apuntan todos en una misma dirección: la independencia del jurisprudente romano, como profesional del Derecho, por encima de cualquier condicionamiento de escuela. Parece así confirmarse por el momento la percepción de Bretone cuando afirma que «dentro de las escuelas jurisprudenciales, como dentro de cualquier otra, la fidelidad — cuando se ejercita la razón- nunca es absoluta» ${ }^{19}$.

19 M. BREtone, «Ius controversum nella giurisprudenza classica», Atti dell'Accademia nazionale dei Lincei, vol. 23 (2008), p. 793. 